\title{
Quantum Hall phases of two-component bosons
}

\author{
T. Graß, ${ }^{1}$ D. Raventós, ${ }^{2}$ M. Lewenstein, ${ }^{1,3}$ and B. Juliá-Díaz ${ }^{1,2}$ \\ ${ }^{1}$ ICFO-Institut de Ciències Fotòniques, Parc Mediterrani de la Tecnologia, 08860 Barcelona, Spain \\ ${ }^{2}$ Departament d'Estructura i Constituents de la Matèria, Universitat de Barcelona, 08028 Barcelona, Spain \\ ${ }^{3}$ ICREA-Institució Catalana de Recerca i Estudis Avançats, 08010 Barcelona, Spain \\ (Received 17 October 2013; revised manuscript received 21 December 2013; published 13 January 2014)
}

\begin{abstract}
The recent production of synthetic magnetic fields acting on electroneutral particles, such as atoms or photons, has boosted interest in the quantum Hall physics of bosons. Adding pseudospin $1 / 2$ to the bosons greatly enriches the scenario, as it allows them to form an interacting integer quantum Hall (IQH) phase with no fermionic counterpart. Here we show that, for a small two-component Bose gas on a disk, the complete strongly correlated regime, extending from the integer phase at filling factor $v=2$ to the Halperin phase at filling factor $v=2 / 3$, is well described by composite fermionization of the bosons. Moreover we study the edge excitations of the IQH state, which, in agreement with expectations from topological field theory, are found to consist of forward-moving charge excitations and backward-moving spin excitations. Finally, we demonstrate how pair-correlation functions allow one to experimentally distinguish the IQH state from competing states, such as non-Abelian spin singlet (NASS) states.
\end{abstract}

DOI: 10.1103/PhysRevB.89.045114

PACS number(s): 73.43.-f, 67.85.-d

\section{INTRODUCTION}

Recent progress in producing strong synthetic gauge fields in neutral systems such as atomic quantum gases [1,2] or photonic fluids [3] has catalyzed research into bosonic quantum Hall states. While in the fractional quantum Hall (FQH) regime the bosonic states are often simply the counterparts of fermionic states, a significant difference occurs for noninteracting particles: Instead of forming an IQH liquid as fermions do, the bosons' fate is to condense. However, as has been strikingly predicted by effective field theory [4,5], this does not exclude the possibility of an IQH phase of bosons. This phase can be obtained in a two-component system at filling factor $v=2$. As has been confirmed by numerical studies [6-8], repulsive two-body contact interaction favors this phase against competing FQH phases. In contrast to the fermionic case, interactions are a crucial ingredient for the integer quantum Hall physics of bosons.

Different from FQH states, integer quantum Hall states have no anyonic excitations, nor do they exhibit topological degeneracies in nontrivial geometries (e.g., tori). Nevertheless, they possess topologically protected edge states which, due to Wen's edge-bulk correspondence [9], make them distinct to conventional bulk insulators. A particularly appealing property of the edge in spin-singlet systems is the fact that it can be excited in twofold ways: by spinless charge excitations ("holons") or by charge-neutral spin excitations ("spinons") [10]. For the edge of the bosonic IQH phase, a $K$-matrix description predicts opposite velocities for these two types of excitations [5], as a consequence of one positive and one negative eigenvalue of the $K$ matrix. This interesting property has been discussed before for a FQH state of spin-1/2 fermions at $v=2 / 3$ in a singlet $[11,12]$.

In the context of FQH physics, two-component Bose gases have been considered in a torus geometry $[13,14]$, where ground state degeneracies suggest them as a candidate for realizing non-Abelian spin singlet (NASS) phases [15]. Quantum many-body states with non-Abelian excitations are particularly relevant, as their use for topological quantum computations has been proposed [16]. A recent study of two-component bosons in a spherical geometry [7], however, gave rise to some controversy: Competitors of the NASS states are the composite fermion (CF) states which have Abelian topological order.

In this article we shed further light on the quantum Hall physics of two-component bosons by performing a systematic numerical study in a disk geometry. After briefly introducing different trial wave functions, we study for $N=6$ particles all incompressible states on the Yrast line, starting with the IQH state at $L_{z}=9$ (in units of $\hbar$ ) and ending with the Halperin state at $L_{z}=21$, where the system is able to fully avoid contact interaction. We find all the incompressible states to be well described by the CF approach [17]. We then study (for $N=8$ ) the edge excitations of the IQH phase. Apart from some exceptions in the forward-moving branch, we find number and spin of the edge excitations to precisely agree with the predictions from effective theory. A model of the edge states based on CF theory is found to accurately describe the wave functions of the backward-moving states. It is shown that the forward-moving states can be modeled by multiplying the ground state wave function with symmetric polynomials. Finally, we demonstrate how pair-correlation functions distinguish the IQH state from competing states in an experiment.

\section{SYSTEM AND TRIAL WAVE FUNCTIONS}

We study a system of $N$ two-component bosons described by the Hamiltonian

$$
H=\sum_{i}^{N} \frac{\left[p_{i}-A\left(z_{i}\right)\right]^{2}}{2 m}+\frac{m}{2} \omega^{2}\left|z_{i}\right|^{2}+V_{0} \sum_{i<j} \delta\left(z_{i}-z_{j}\right)
$$

where $z_{i}=x_{i}+i y_{i}$ represents the position of the boson, $A(z)=\frac{B}{2}(x,-y)$ is a gauge potential, and $m, V_{0}$, and $\omega$ are positive parameters specifying the mass, the two-body 
interaction strength, and the frequency of a harmonic confinement. The single-particle part of $H$ has a Landau level (LL) structure, and is solved by Fock-Darwin (FD) states $\varphi_{n, \ell}$, which in the lowest Landau level (LLL) $\operatorname{read} \varphi_{0, \ell}(z) \propto$ $z^{\ell} \exp \left(-|z|^{2} / 4\right)$, in units of length given by $\lambda=\sqrt{\hbar /\left(M \omega_{0}\right)}$, and $\omega_{0} \equiv \sqrt{\omega^{2}+\frac{B^{2}}{4 m^{2}}}$.

One way to account for interactions is the CF theory developed by Jain [17]. It provides a compelling picture to understand both IQH and FQH phases on an equal footing: By attaching magnetic fluxes to each particle, one obtains CFs, which are assumed to behave like noninteracting particles; that is, they fill one or several LLs. Originally, this theory was formulated for fermions, but it has been extended to bosonic quantum Hall phases triggered by the experimental progress in producing synthetic gauge fields acting on ultracold atoms [18]. Recently, CF states for two-component Bose systems have been introduced and studied in a spherical geometry [7].

Here we consider a system on a disk, for which a similar $\mathrm{CF}$ construction is detailed in the Appendix. Omitting the omnipresent Gaussian factor, the wave functions at angular momentum $L_{z}$ read

$$
\Psi_{L_{z}}^{\left[n_{a}, n_{b}\right]}=\mathcal{P}_{\mathrm{LLL}}\left[\Phi_{n_{a}}\left(\left\{z_{a}\right\}\right) \Phi_{n_{b}}\left(\left\{z_{b}\right\}\right) J_{1}(\{z\})\right] .
$$

The last term is a Jastrow factor $J_{1}(\{z\})=\prod_{i<j}\left(z_{i}-z_{j}\right)$, which attaches one magnetic flux to each particle, turning the bosons into CFs. The wave function of the composite particles is given by the Slater determinants $\Phi_{n_{a}}$ and $\Phi_{n_{b}}$, for particles of type $a$ and $b$, respectively. The indices $n_{a(b)}$ yield the number of LLs occupied by the CFs. If $n_{a}=n_{b}$, the total spin is zero, $S=0$. Importantly, negative $n_{a}$ and $n_{b}$ shall refer to flux-reversed LLs: $\Phi_{-n} \equiv \Phi_{n}^{*}$. Finally, $\mathcal{P}_{\text {LLL }}$ projects back into the LLL of the bosonic system. We perform this projection in the standard way by replacing conjugate variables $z^{*}$ by derivatives $\partial_{z}$.

The only difference between Eq. (2) and the corresponding definition on a sphere is the fact that in closed geometries the number of states in each LL is finite. This gives rise to the notion of "completely filled" LLs, and the state $\Psi^{\left[n_{a}, n_{b}\right]}$ is uniquely defined. Depending on the sign of $n=n_{a}+$ $n_{b}$, its filling factor is $v_{ \pm}=n /(n \pm 1)$. Contrarily, on a disk, there is more than one way to distribute $N_{a}\left(N_{b}\right)$ particles in $n_{a}\left(n_{b}\right)$ LLs. Typically each choice leads to a different total angular momentum $L_{z}$, such that wave functions at different angular momentum $L_{z}$ correspond to the same filling factor $v$ in the thermodynamic limit. Note that, for $\left|n_{a}\right|=\left|n_{b}\right|=1$, however, the wave functions are unique also on a disk. In particular, $\Psi^{[-1,-1]}$ has $L_{z}=N^{2} / 4$ and corresponds to an integer filling factor, $v=2$. In contrast to all other $\mathrm{CF}$ wave functions with fractional filling, this wave function might describe an IQH liquid.

Another important trial wave function, obtained within the CF theory by putting all composite particles to the LLL $\left(\Psi^{[1,1]}\right)$, is the Halperin state [19], explicitly given by

$$
\Psi_{\mathrm{H}} \sim \prod_{i<j}\left(z_{i a}-z_{j a}\right)^{2} \prod_{i<j}\left(z_{i b-} z_{j b}\right)^{2} \prod_{i, j}\left(z_{i a}-z_{j b}\right) .
$$

It is a spin singlet wave function at filling $v=2 / 3$, with zero energy in a two-body contact potential, and describes an Abelian FQH phase. A series of non-Abelian quantum Hall

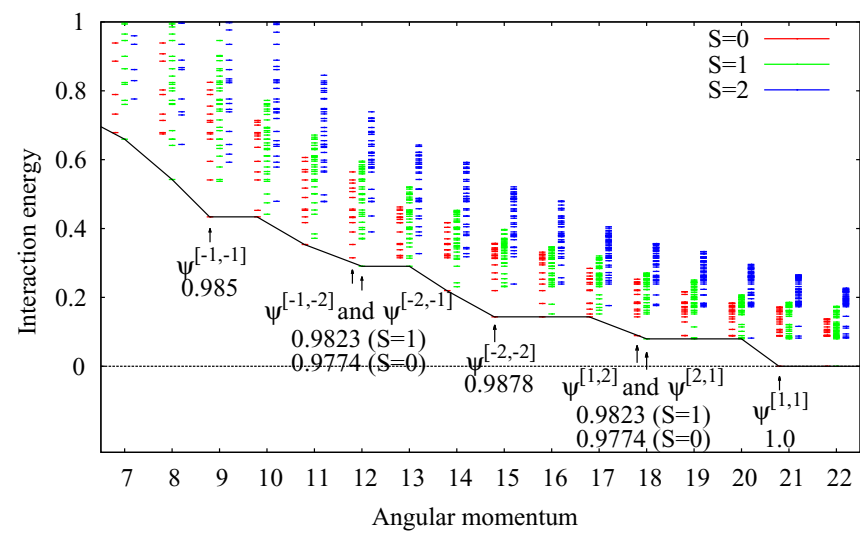

FIG. 1. (Color online) Yrast line for $N=6$ : For the incompressible states (marked by arrows) we give the overlap with corresponding $\mathrm{CF}$ wave functions.

states can be constructed from it by forming $k$ clusters, putting each cluster into a Halperin state, and symmetrizing over all possible clusterizations [15]. In this way, one obtains the NASS states at filling factor $v=2 k / 3$ as the zero-energy eigenstates of $(k+1)$-body contact interaction.

\section{YRAST LINE}

We have studied $N=6$ two-component bosons in the LLL on a disk by exactly diagonalizing the SU(2)-symmetric two-body contact interaction. The presence of an additional harmonic trapping in $H$ which is invariant under spatial rotations along the $z$ axis and under spin rotations will not modify the eigenstates of the system, but simply increase the energy eigenvalues by a value proportional to $L_{z}$. Properly choosing the trapping frequency, one can tune the ground state of the system to different $L_{z}$.

The system's Yrast line (i.e., the spectrum of the interaction energy at fixed $L_{z}$ ), is shown in Fig. 1. Different $L_{z}=$ $9,12,15,18,21$ correspond to incompressible states, that is, here an increase of angular momentum will not (directly) lead to a decrease in energy. Notably, for all these $L_{z}$ it is possible to construct $\mathrm{CF}$ states. Moreover, exact ground states and CF states agree in spin, and have very good overlap (>0.97). At $L_{z}=21$, the overlap equals 1 , as the Halperin state of Eq. (3) becomes the exact ground state. At $L_{z}=18$, two CF states with $S_{z} \equiv\left(N_{a}-N_{b}\right) / 2=0$ can be constructed: $\Psi^{[1,2]}$ and $\Psi^{[2,1]}$. Accordingly, the ground state is a triplet, but notably, also the antisymmetric combination of the two states gives rise to a quasidegenerate singlet state. For $L_{z}=15$, the CF construction yields a unique singlet phase, $\Psi^{[-2,-2]}$, with overlap 0.9878 and large gap. For $L_{z}=12$, the situation is similar to $L_{z}=18$, with a triplet ground state and a quasidegenerate singlet state obtained from two possible $\mathrm{CF}$ states, $\Psi^{[-1,-2]}$ and $\Psi^{[-2,-1]}$. The incompressible phase with smallest $L_{z}$ is found for $L_{z}=9$ : the clearly gapped ground state is a singlet and has large overlap $(0.985)$ with $\Psi^{[-1,-1]}$.

\section{EDGE PHYSICS OF THE IQH PHASE}

We now focus on this lowest- $L_{z}$ state on the Yrast line, for which we can extend our numerical study to $N=8$ particles 
TABLE I. Number of modes of $H_{\text {edge }}$ with $v_{s}<0$ and $v_{c}>0$.

\begin{tabular}{lcccccccc}
\hline \hline$\Delta L_{z}$ & -4 & -3 & -2 & -1 & +1 & +2 & +3 & +4 \\
\hline Number of singlets & 2 & 1 & 1 & 0 & 1 & 2 & 3 & 5 \\
Number of triplets & 2 & 2 & 1 & 1 & 0 & 0 & 0 & 0 \\
Number of quintets & 1 & 0 & 0 & 0 & 0 & 0 & 0 & 0
\end{tabular}

and, accordingly, $L_{z}=16$. Compared to $N=6$, we find an only slightly smaller overlap, $\left|\left\langle\mathrm{GS} \mid \Psi^{[-1,-1]}\right\rangle\right|=0.9709$. As $\Psi^{[-1,-1]}$ describes a spin singlet with integer filling $v=2$, and the phase turns out to be strongly gapped and incompressible, all prerequisites for an IQH phase are fulfilled. Previous studies provided evidence of the integer topological character of this phase by analyzing spectral properties [6-8], and wave functions on a sphere [7]. In the present paper, we consider the equivalent system in a plane, and focus on the physics at the edge to characterize its topology [9].

An effective theory of the edge physics in fermionic singlet states [11] is applicable also to the bosonic IQH state. It allows for a straightforward counting of the edge excitations. This theory is based on the observation that edge excitations of a spin singlet state might either be excitations which change angular momentum of the spin-up (down) particles, or be excitations which flip the spin of some particles. Thus, the effective edge Hamiltonian has the form [11]

$$
H_{\text {edge }} \propto v_{s}\left(S_{z}^{2}+\sum_{l} l b_{l}^{\dagger} b_{l}\right)+v_{c} \sum_{l} l c_{l}^{\dagger} c_{l} .
$$

Here, the first term denotes the spinon excitations with velocity $v_{s}$, and the second term the holon excitations with velocity $v_{c}$. The operators $b_{l}$ and $c_{l}$ annihilate bosonic modes at angular momentum $l$.

For a counting of the modes, we only consider pure charge excitations $\left(\left\langle b_{l}^{\dagger} b_{l}\right\rangle=0\right)$ or pure spin excitations $\left(\left\langle c_{l}^{\dagger} c_{l}\right\rangle=0\right)$, as mixed charge/spin excitations are expected at higher energies. Moreover, we assume that $v_{c}>0$ and $v_{s}<0$, such that the charge (spin) excitations are located at positive (negative) $\Delta L_{z}$. As a result, we find the multiplicities listed in Table I: The spinless $c$ modes are simply counted by the number of positive-integer sums which add up to $\Delta L_{z}$. Since $S_{z}^{2}=0$ for all $c$ modes, they must be singlets. In the spinon branch, instead, we have to count the number of positive-integer sums which add up to $\left|\Delta L_{z}\right|-S_{z}^{2}$, where $S_{z}$ now might take also nonzero integer values. For example, for $\Delta L_{z}=-1$, we might have $S_{z}= \pm 1$ and $\left\langle b_{l}^{\dagger} b_{l}\right\rangle=\delta_{l 1}$, or $S_{z}=0$ and $\left\langle b_{l}^{\dagger} b_{l}\right\rangle=0$. These three states yield a triplet. Following this reasoning, the first occurrence of a quintet $(S=2)$ is expected for $\Delta L_{z}=-4$.

\section{BACKWARD-MOVING EDGE STATES}

In the spectrum shown in Fig. 2, we find one gapped triplet ground state at $\Delta L_{z}=-1$, and two quasidegenerate gapped ground states, one singlet and one triplet, at $\Delta L_{z}=-2$. This perfectly matches with the counting expected from effective theory. Also at $\Delta L_{z}=-3$ and $\Delta L_{z}=-4$, the spin of the lowest states agrees with the spin predicted by effective theory,

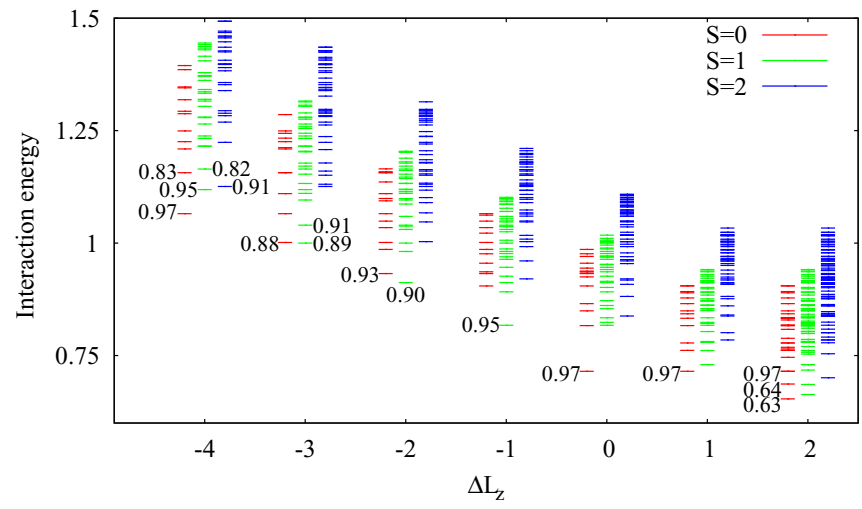

FIG. 2. (Color online) Low-energy part of the spectrum of $H$, obtained by exact diagonalization of a system with $N=8$ around $L_{z}=16$. The numbers beside some states denote their overlap with the trial wave functions described in the text.

but the degeneracy lifting within the ground state manifold becomes larger than the gap to the excited states. A particularly striking confirmation of the effective theory is the fact that at $\Delta L_{z}=-4$ a $S=2$ multiplet becomes member of the ground state manifold.

A simple intuitive explanation for the presence of a backward-moving state, which directly leads to a scheme for constructing trial wave functions, can be given in terms of the CF approach: Since the ground state, $\Psi^{[-1,-1]}$, describes an IQH phase of CFs which are subjected to a flux-reversed magnetic field, forward-directed edge excitations of the CFs constitute backward-directed edge excitations of the bosons. More formally, as a consequence of the complex conjugation of the Slater determinants in $\Psi^{[-1,-1]}$, the edge excitation of the CFs (that is the shift of one or several CFs to higher angular momentum) will correspond to a reduced angular momentum of the bosons.

Following this reasoning, we have constructed trial wave functions for edge states with $-4 \leqslant \Delta L_{z} \leqslant-1$. For example, consider the state with $S_{z}=0$ at $\Delta L_{z}=-1$ : The ground state $\Psi^{[-1,-1]}$ consists of four spin-up and four spin-down CFs, each filling the FD states with $\ell=0, \ldots, 3$ in the flux-reversed LLL. An edge state can then be obtained in two ways: for either the spin-up or the spin-down $\mathrm{CFs}$, we replace the FD state with $\ell=3$ by a FD state with $\ell=4$, which after complex conjugation leads to $\Delta L_{z}=-1$. Strikingly, after projecting these wave functions into the LLL, both choices lead to exactly the same wave function, and we recover a single state at $S_{z}=0$, as demanded by both the effective theory and the numerical results. This becomes more remarkable for $\Delta L_{z}<-1$ : At $\Delta L_{z}=-2$, we find five ways to construct $S_{z}=0$ edge states, but they reduce to two linearly independent states. At $\Delta L_{z}=-3$, ten different constructions lead to three states, and at $\Delta L_{z}=-4$ twenty constructions yield precisely five different states. Thus, the CF construction is in perfect agreement with the counting of modes. Apart from the counting, also the overlaps of the trial states with the exact states are remarkably high. They are explicitly given within Fig. 2, and for any of the eleven edge states in the interval $-4 \leqslant \Delta L_{z} \leqslant-1$ they are larger than 0.82 , demonstrating the power of the $\mathrm{CF}$ description. 

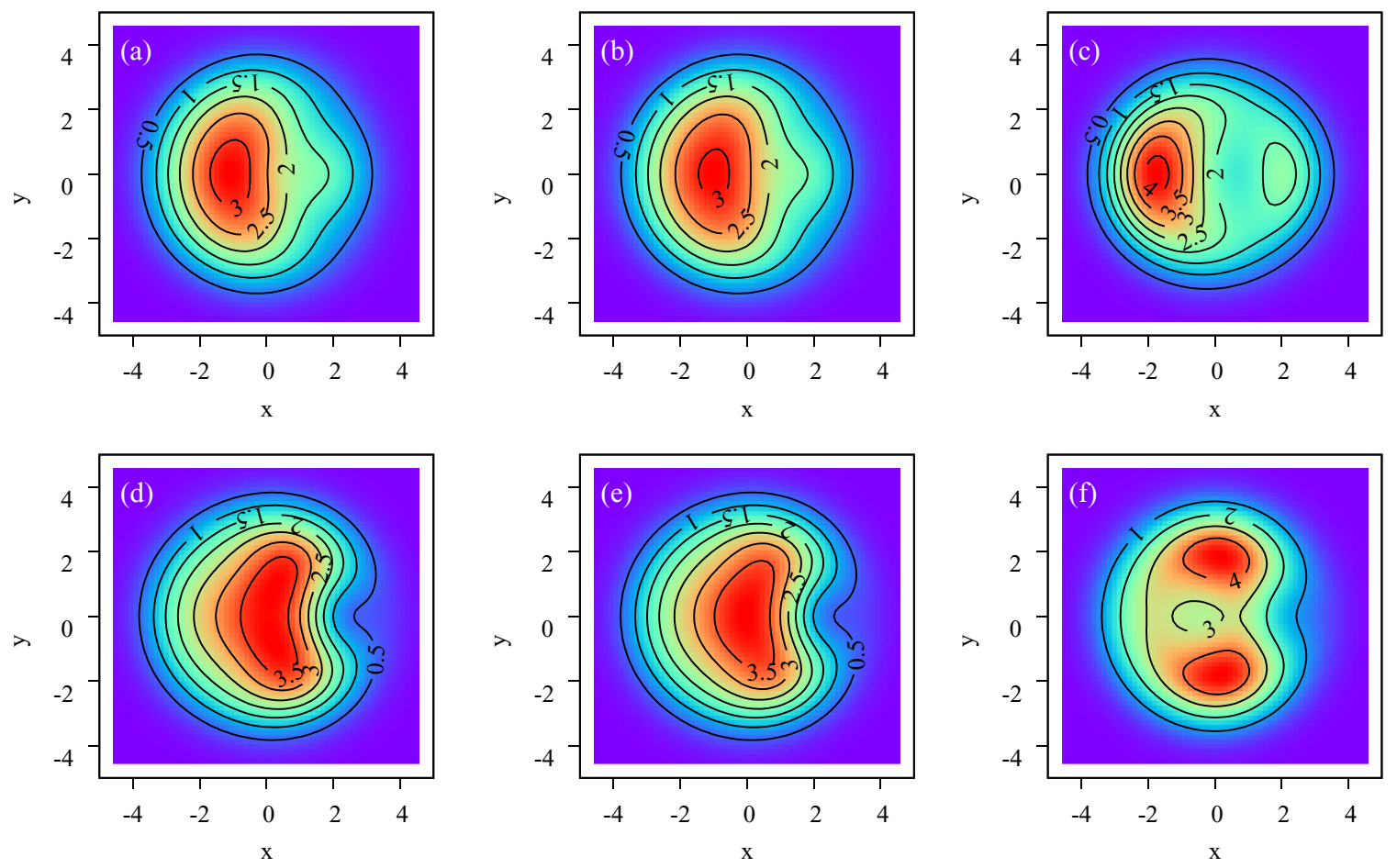

FIG. 3. (Color online) Spin-dependent pair-correlation function for $N=8$ particles, showing the probability density of finding one particle with given spin after fixing another particle of the same spin [(a)-(c)] or of opposite spin [(d)-(f)] to $(x, y)=(2.5,0)$. In plots (a) and (d), the system is in the exact ground state at $L_{z}=16$. In (b) and (e), the system is in the corresponding CF state, and in (c) and (f) it is in the $v=4 / 3$ NASS phase.

\section{FORWARD-MOVING EDGE STATES}

For $\Delta L_{z}>0$, the effective theory predicts spin singlet ground states, with degeneracy $1,2,3,5, \ldots$ for $\Delta L_{z}=$ $1,2,3,4, \ldots$ Indeed we find a single singlet ground state at $\Delta L_{z}=1$, though it is not separated by a large gap from a second, low-lying triplet state; see Fig. 2. Also at $\Delta L_{z}=2$ there is a singlet ground state, but a nearby second state in the spectrum is a triplet state, instead of a second spin singlet. At $\Delta L_{z}=3$, even the ground state is a triplet. It has been argued that forward-moving edge states have a large velocity and thus merge with bulk excitations, spoiling the spectral structure expected from effective theory [7,11]. Moreover, we note that the state $\Psi^{[-1,-1]}$ is the first incompressible state on the Yrast line. Therefore, while backward-moving modes of this state do not interfere with forward-moving edge modes of other incompressible states, the forward-moving excitations of $\Psi^{[-1,-1]}$ are expected to mix with backwardmoving modes of an incompressible triplet phase at $L_{z}=20$ (for $N=8$ ).

Nevertheless, it is possible to identify some states in the spectrum of Fig. 2 as forward-moving edge states of $\Psi^{[-1,-1]}$. We construct them by multiplying the ground state by homogeneous polynomials which are symmetric in all variables. Such excitation might either act on the bosons, that is on the wave function $\Psi^{[-1,-1]}$, or on the composite fermions, that is on the CF wave function before LLL projection. Remarkably, the latter approach yields slightly better results.

For $\Delta L_{z}=1$, the construction yields one singlet, having overlap 0.9709 with the exact state. Note that this is precisely the overlap of the exact ground state at $\Delta L_{z}=0$ with $\Psi^{[-1,-1]}$, suggesting that the construction of the edge itself is exact, and the slight deviation of the overlap from unity is caused by a discrepancy between the ground state at $L_{z}=16$ and the CF state. Also, the ground states at both $\Delta L_{z}=0$ and $\Delta L_{z}=1$ have exactly the same energy.

At $\Delta L_{z}=2$, the energy of only the sixth state in the spectrum, a singlet, matches with the ground state energy at $\Delta L_{z}=0$. This state is well reproduced (again overlap 0.9709) by our construction of edge states which now yields two singlet states. At lower energies, we find two singlet states, two triplet states, and one $S=2$ multiplet. Each of the two singlet states has an overlap around 0.63 with our edge state construction, suggesting that a linear combination of the two states would reasonably well agree. In that way, we can, out of the three low-energy singlet states, recognize two as the edge states predicted by effective theory.

\section{EXPERIMENTAL REALIZATION}

We propose to use a system of two-component Bose atoms subject to two independent strong synthetic fields, employing the method of Ref. [20]. To this aim one can use two microwave couplings of opposite circular polarization to couple, for instance, $F=1, M= \pm 1$ and $F=2, M= \pm 2$ states of ${ }^{87} \mathrm{Rb}$ (employing methods proposed in Ref. [21] and pioneered for spin-dependent lattices in Refs. [22,23]), or $F=3, M= \pm 3$ and $F=4, M= \pm 4$ states of ${ }^{133} \mathrm{Cs}$, as realized recently in Ref. [24]. The proposed scheme could be tuned from the microscopic regime to the macroscopic regime, where it could resolve a competition between NASS and the 
CF state at $v=4 / 3$ in the thermodynamic limit [7], or for $N \geqslant 16$, nonaccessible by exact diagonalization. The favored phase could be detected by a measurement of correlations, which has been demonstrated with single-atom resolution in Refs. [25,26]. Here, the same method can be used by switching on rapidly a deep lattice to localize the atoms.

To illustrate how correlations may identify the phase, we refer to a different competition which takes place for $N=8$ at $L_{z}=16$ : Here the CF picture with the $v=2$ state describes well the ground state (overlap 0.97), but an alternative trial wave function is the $v=4 / 3$ NASS state (overlap 0.52). Note that the CF state and the NASS state themselves have overlap 0.41 , despite their different topological order. The overlaps certainly give a clear picture in favor of the CF state, but they are not accessible to experiment. Instead, measuring the paircorrelation functions allows us to distinguish clearly between CF and NASS states, as shown in Fig. 3.

\section{CONCLUSIONS}

We have studied quantum Hall phases of two-component bosons on a disk. All incompressible phases are understood in the CF picture. The edge states identify the IQH phase of bosons. This phase could be realized in experiments with cold atoms, and detected by measuring pair-correlation functions.

\section{ACKNOWLEDGMENTS}

We thank Andrea Alberti for helpful discussions. This work has been supported by the EU (SIQS, EQUAM), ERC (QUAGATUA), Spanish MINCIN (FIS2008-00784 TOQATA), Generalitat de Catalunya (2009-SGR1289), and Alexander von Humboldt Stiftung. B.J.D. is supported by the Ramón y Cajal program.

\section{APPENDIX: COMPOSITE FERMION CONSTRUCTION}

A quite general feature of quantum Hall wave functions is strong anticorrelations between the particles, introduced by the magnetic fluxes. Formally, such anticorrelations are described by a Jastrow factor,

$$
J_{m}(\{z\})=\prod_{i<j}\left(z_{i}-z_{j}\right)^{m},
$$

which prohibits two particles being at the same position $z=x+i y$. In the composite fermion picture [17], such Jastrow factors are interpreted as flux attachments; that is, $J_{m}$ makes each particle be seen by the other particles as a vortex with vorticity $m$. A system of $N$ particles and $N_{\Phi}$ magnetic fluxes can thus alternatively be seen as a system of $N$ composite particles, and $N_{\Phi}^{\prime} \equiv N_{\Phi}-m N$ fluxes. For the exchange symmetry of the composite particles to be fermionic, $m$ has to be odd (even) if the original particles are bosons (fermions). The appeal of the composite fermion picture is based on the fact that the composite particles very often turn out to form integer quantum Hall liquids; that is, their wave functions are simply given by a Slater determinant for a system with $N_{\Phi}^{\prime}$ fluxes.

The composite fermion construction is often carried out for systems on the surface of a sphere. Such closed geometry offers the advantage that each Landau level has a finite number of states, which defines the notion of "filled" Landau levels. Each level then accounts for a well defined, finite amount of magnetic flux, and the number of occupied Landau levels is uniquely defined by $N_{\Phi}^{\prime}$. Accordingly, one can also define a filling factor, $v=N / N_{\Phi}$, and in this way conveniently relate the system in the closed geometry to a thermodynamically large system in a plane geometry.

In this paper, we study a small system in a plane geometry. For a rotationally symmetric system, it then becomes most convenient to introduce the angular momentum $L_{z}$ (in the direction perpendicular to the system in the $x y$ plane) as a constant of the motion. By assuming that the system spends all angular momentum as relative angular momentum between pairs of particles, $L_{z}$ (in units of $\hbar$ ) relates to the number of fluxes as

$$
N_{\Phi}=\frac{L_{z}}{\frac{1}{2}(N-1)} .
$$

This formula allows to relate the filling factor $v$ to the angular momentum $L_{z}$, but it fails in the presence of angular momentum with respect to the center of mass. Since centerof-mass angular momentum scales linearly with $N$, whereas the relative angular momentum scales with the number of pairs, that is with $N^{2}$, Eq. A2 holds, for arbitrary states, in the thermodynamical limit.

To perform the composite fermion construction, we note that the Jastrow term $J_{m}$ consumes $\frac{m}{2} N(N-1)$ quanta of angular momentum. Accordingly, a state with quantum number $L_{z}$ is described by the Jastrow term multiplied with a Slater determinant of total angular momentum $L_{z}^{\prime}=$ $L_{z}-\frac{m}{2} N(N-1)$. It may happen that $L_{z}^{\prime}<0$, a situation in which more fluxes have been attached to the particles than the magnetic field provides. Effectively, the composite particles then feel an opposite magnetic field. Such flux reversion corresponds to a complex conjugation of the wave function.

For distributing the angular momentum $L_{z}^{\prime}$ over $N$ composite fermions, we have to note that the Landau level structure implies that $\ell \geqslant-n$, where $\ell$ is the angular momentum, and $n$ is the energy quantum number of the single-particle states. That is, in the lowest Landau level $(n=0)$, we have states with $\ell=0,1,2, \ldots$, whereas in higher Landau levels $(n>0)$ also states with negative angular momentum exist. Starting in the lowest Landau level, we fill each single-particle state from $\ell=0$ up to $\ell_{\max }^{(1)}$, and similarly for higher levels from $\ell=-n$ to $\ell_{\max }^{(n)}$. Different from the closed geometry, in the plane system there are no a priori values for the maximum angular momentum $\ell_{\max }^{(n)}$ in each Landau level. However, changing $\ell_{\max }^{(n)}$ will typically also modify the total angular momentum $L_{z}^{\prime}$ of the Slater determinant. Accordingly, specifying the angular momentum will fix this freedom.

We now turn to the two-component case discussed in this paper. In the main text, we have introduced the composite fermion wave functions as

$$
\Psi_{L_{z}}^{\left[n_{a}, n_{b}\right]}=\mathcal{P}_{\mathrm{LLL}}\left[\Phi_{n_{a}}\left(\left\{z_{a}\right\}\right) \Phi_{n_{b}}\left(\left\{z_{b}\right\}\right) J_{1}(\{z\})\right],
$$

for a given $L_{z}$, and given numbers $N_{a}$ and $N_{b}$ of type- $a$ and type- $b$ particles (or pseudospin). In the Jastrow term $J_{1}(\{z\})$, no distinction between $a$ and $b$ particles is made. After the flux attachment, however, the two components should be independent, so we write $\left.\Phi_{n_{a}}\left\{z_{a}\right\}\right)\left(\Phi_{n_{b}}\left(\left\{z_{b}\right\}\right)\right.$ for 
TABLE II. All composite fermion wave functions for $N_{a}=N_{b}=3$ on a disk. For each angular momentum $L_{z}$, at which a composite fermion construction exists, we give the corresponding angular momentum $L_{z}^{\prime}$ of the composite fermions, and list all possible ways of occupying the composite fermion Landau levels.

\begin{tabular}{lrrll}
\hline \hline Wave function & $L_{z}$ & $L_{z}^{\prime}$ & Single-particle states occupied by $a$-type CF & Single-particle states occupied by $b$-type CF \\
\hline$\Psi_{9}^{[-1,-1]}$ & 9 & -6 & LLL: $\ell=0,1,2$ (flux-reversed) & LLL: $\ell=0,1,2$ (flux-reversed) \\
$\Psi_{12}^{[-2,-1]}$ & 12 & -3 & LLL: $\ell=0,1 ; 1$ LL: $\ell=-1$ (flux-reversed) & LLL: $\ell=0,1,2$ (flux-reversed) \\
$\Psi_{12}^{[-1,-2]}$ & 12 & -3 & LLL: $\ell=0,1,2$ (flux-reversed) & LLL: $\ell=0,1 ; 1 \mathrm{LL}: \ell=-1$ (flux-reversed) \\
$\Psi_{15}^{[2,2]}$ & 15 & 0 & LLL: $\ell=0,1 ; 1 \mathrm{LL}: \ell=-1$ & LLL: $\ell=0,1 ; 1 \mathrm{LL}: \ell=-1$ \\
$\Psi_{18}^{[2,1]}$ & 18 & 3 & LLL: $\ell=0,1 ; 1 \mathrm{LL}: \ell=-1$ & LLL: $\ell=0,1,2$ \\
$\Psi_{18}^{[1,2]}$ & 18 & 3 & LLL: $\ell=0,1,2$ & LLL: $\ell=0,1 ; 1 \mathrm{LL}: \ell=-1$ \\
$\Psi_{21}^{[1,1]}$ & 21 & 6 & LLL: $\ell=0,1,2$ & LLL: $\ell=0,1,2$ \\
\hline \hline
\end{tabular}

the Slater determinant of the $a(b)$ particles. The indices $n_{a}$ $\left(n_{b}\right)$ shall determine the number of occupied Landau levels in both Slater determinants, and negative values of $n_{a}\left(n_{b}\right)$ shall denote the case of reversed flux. While in a closed geometry, this information would already uniquely define the wave function, the plane geometry, as explained above, allows for different ways of distributing $N_{a}\left(N_{b}\right)$ particles to $n_{a}\left(n_{b}\right)$ Landau levels. But again, the total angular momentum $L_{z}$ can be used as an additional label.

Trying to make a connection to the thermodynamic limit and/or the system on the sphere, one associates the wave function $\Psi_{L_{z}}^{\left[n_{a}, n_{b}\right]}$ with a filling factor fully defined by the choice of $\left[n_{a}, n_{b}\right]$. It reads [7]

$$
v=\frac{n_{a}+n_{b}}{n_{a}+n_{b}+1} .
$$

There is one possibility for an integer filling factor, and thus an integer quantum Hall phase, at $n_{a}=n_{b}=-1$, and $v=2$. For this state, $\Psi^{[-1,-1]}$, it is possible to write down a closed expression for the angular momentum $L_{z}$ : The Jastrow factor $J_{1}$ provides $\frac{1}{2} N(N-1)$ quanta of angular momentum. Both components fill $\mathrm{N} / 2$ states in the flux-reversed lowest Landau level, yielding $L^{*}=-\sum_{\ell=0}^{N / 2-1} \ell=-\frac{N}{8}(N-2)$ quanta of angular momentum per component. This yields a total angular momentum $L_{z}=\frac{1}{2} N(N-1)+2 L^{*}=\frac{N^{2}}{4}$.
For more general cases, we exemplify the construction on the plane by listing all possible composite fermion wave functions with $N_{a}=N_{b}=3$ in Table II. There, we give the corresponding angular momentum of the system and the composite fermions, $L_{z}$ and $L_{z}^{\prime}$, and we specify which single-particle states are occupied by the composite particles. These wave functions yield all incompressible phases found on the Yrast line shown in Fig. 1 of the main part. While the choice $N_{a}=N_{b}$ implies that the $z$ component of spin $S_{z}$ is zero, the total spin is given by the multiplicities of the composite fermion wave functions. For $n_{a}=n_{b}$, there is a single wave function and thus a singlet, whereas for $n_{a} \neq n_{b}$ (at least) two equivalent wave functions related through a spin rotation exist. The composite fermion construction then yields an SU(2) multiplet.

An important part in the definition of Eq. (A3) is the projection operator $\mathcal{P}_{\text {LLL }}$ which shall bring the expression in the lowest Landau level, that is, the low-energy Hilbert space of the problem. Projecting in the lowest Landau level amounts to getting rid of complex conjugate variables $z^{*}$, which naturally occur when the Slater determinants extend to higher Landau levels. While there is no unique way of performing the projection, a natural way [17] is to replace the complex conjugate coordinate $z^{*}$ by a derivative $\partial / \partial z$, leaving the total angular momentum of the wave function unchanged.
[1] J. Dalibard, F. Gerbier, G. Juzeliūnas, and P. Öhberg, Rev. Mod. Phys. 83, 1523 (2011).

[2] N. Goldman, G. Juzeliūnas, P. Öhberg, and I. Spielman, arXiv:1308.6533.

[3] I. Carusotto and C. Ciuti, Rev. Mod. Phys. 85, 299 (2013).

[4] Y.-M. Lu and A. Vishwanath, Phys. Rev. B 86, 125119 (2012).

[5] T. Senthil and M. Levin, Phys. Rev. Lett. 110, 046801 (2013).

[6] S. Furukawa and M. Ueda, Phys. Rev. Lett. 111, 090401 (2013).

[7] Y.-H. Wu and J. K. Jain, Phys. Rev. B 87, 245123 (2013).

[8] N. Regnault and T. Senthil, Phys. Rev. B 88, 161106(R) (2013).

[9] X.-G. Wen, Int. J. Mod. Phys. B 6, 1711 (1992).

[10] A. Balatsky and M. Stone, Phys. Rev. B 43, 8038 (1991).

[11] J. E. Moore and F. D. M. Haldane, Phys. Rev. B 55, 7818 (1997).

[12] Y.-H. Wu, G. J. Sreejith, and J. K. Jain, Phys. Rev. B 86, 115127 (2012).
[13] T. Graß, B. Juliá-Díaz, N. Barberán, and M. Lewenstein, Phys. Rev. A 86, 021603(R) (2012).

[14] S. Furukawa and M. Ueda, Phys. Rev. A 86, 031604(R) (2012).

[15] E. Ardonne and K. Schoutens, Phys. Rev. Lett. 82, 5096 (1999).

[16] C. Nayak, S. H. Simon, A. Stern, M. Freedman, and S. Das Sarma, Rev. Mod. Phys. 80, 1083 (2008).

[17] J. K. Jain, Composite Fermions (Cambridge University Press, Cambridge, 2007).

[18] N. R. Cooper and N. K. Wilkin, Phys. Rev. B 60, 16279(R) (1999).

[19] B. I. Halperin, Helv. Phys. Acta 56, 75 (1983).

[20] B. Juliá-Díaz, D. Dagnino, K. J. Günter, T. Graß, N. Barberán, M. Lewenstein, and J. Dalibard, Phys. Rev. A 84, 053605 (2011). 
[21] D. Jaksch, H.-J. Briegel, J. I. Cirac, C. W. Gardiner, and P. Zoller, Phys. Rev. Lett. 82, 1975 (1999).

[22] O. Mandel, M. Greiner, A. Widera, T. Rom, T. W. Haensch, and I. Bloch, Nature (London) 425, 937 (2003).

[23] O. Mandel, M. Greiner, A. Widera, T. Rom, T. W. Hänsch, and I. Bloch, Phys. Rev. Lett. 91, 010407 (2003).
[24] M. Genske, W. Alt, A. Steffen, A. H. Werner, R. F. Werner, D. Meschede, and A. Alberti, Phys. Rev. Lett. 110, 190601 (2013).

[25] W. S. Bakr, J. I. Gillen, A. Peng, S. Foelling, and M. Greiner, Nature (London) 462, 74 (2009).

[26] J. F. Sherson, C. Weitenberg, M. Endres, M. Cheneau, I. Bloch, and S. Kuhr, Nature (London) 467, 68 (2010). 